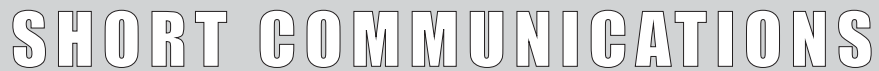

\section{Isolation and antibiotic resistance of Ureaplasma spp. isolated from urogenital specimen between 2002 to 2007}

\author{
Tito Del Gaudio', M.A Distasi', M. Porzio', Giuseppe Miragliotta² \\ 'Laboratorio Analisi P.O. di Andria, AUSL BAT. \\ ${ }^{2}$ Sezione di Microbiologia, Dip. MIDIM, Università degli Studi, Policlinico, P.zza G. Cesare, 4, 70124 - Bari, Italy
}

Key Words: Ureaplasma, urogenital samples, antimicrobial resistance

Isolamento ed antibiotico-resistenza di Ureaplasma spp. isolati da campioni urogenitali nel periodo 2002-2007

\section{SUMMARY}

Ureaplasma spp. and Mycoplasma hominis are frequently isolated from urogenital samples. Ureaplasma spp is responsible for cervicovaginitis, salpingitis, urethritis, epididymitis, male and female infertility, spontaneous abortion, and during pregnancy, for the premature rupture of the membranes, because of chorionamnionitis. Our study aimed to establish the pattern of antimicrobial resistance among Ureaplasma spp isolated in the area of Andria, Apulia Region, from January 2002 to December 2007. 240/78I (30.7\%) of the urogenital samples examined were found Ureaplasma spp.-positive. I52/240 (63.3\%) were >104 UFC/ml and 88/240 (36.7\%) were < $<0^{4}$ UFC/ml. With regard to the resistance rate, we observed significant increase in resistance to ciprofloxacin, ofloxacin, erythromycin, clarithromycin, and azithromycin. While we did not observe resistance to doxycycline, strains resistant to tetracycline, josamycin, and pristinamycins, were isolated during last years of investigation. Our data may help improve the management of these infections above all in consideration of the differences among isolates in different geographic regions.

Received January 27, 2009

Accepted March 9, 2009

\section{INTRODUZIONE}

I micoplasmi sono microrganismi inclusi nella classe Mollicutes, che comprende la famiglia Mycoplasmataceae a cui appartengono due generi responsabili di infezioni umane Mycoplasma e Ureaplasma. A differenza degli altri procarioti, i micoplasmi non possiedono, esternamente alla membrana citoplasmatica, una parete cellulare. L'assenza della parete cellulare conferisce loro alcune caratteristiche peculiari, in particolare la capacità di assumere forme diverse in base alle condizioni nutritive e alla pressione osmotica del mezzo in cui vivono e la resistenza agli antibiotici beta-lattamici che inibiscono la sintesi della parete cellulare. Ureaplasma spp. e Mycoplasma hominis sono frequentemente isolati da campioni urogenitali. Purchè con carica $>10^{4} \mathrm{UFC} / \mathrm{ml}$, questi microrganismi possono essere responsabili di varie patologie sia nei soggetti di sesso femminile (cervicovaginite, salpingite) che in quelli di sesso maschile (uretrite, epididimite). Ureaplasma spp. è inoltre associato a infertilità sia maschile che femminile nonché ad aborto spontaneo ed a rottura precoce delle membrane in corso di gravidanza conseguente a corionamnionite. Nel nostro lavoro abbiamo valutato l'incidenza di Ureaplasma spp. e la relativa antibioticoresistenza nell'area della città di Andria, Regione Puglia.

\section{MATERIALI E METODI}

Nel periodo gennaio 2002 - dicembre 2007 sono stati esaminati 781 campioni urogenitali: 635 tamponi vaginali, 107 liquidi seminali, 33 tamponi uretrali, 6 urine. Per la ricerca di Uu ed il relativo antibiogramma è stato utilizzato il sistema bioMérieux "Mycoplasma IST" da gennaio 2002 a ottobre 2003 e "Mycoplasma IST 2" da novembre 2003 a dicembre 2007. Con questo metodo viene associato un brodo di coltura ad una galleria costituita da 16 test per "Mycoplasma IST" e da 22 test "Mycoplasma

\section{Corresponding author: Tito Del Gaudio}


IST 2", che permettono l'identificazione, la conta indicativa di Ureaplasma spp. (Ureaplasma parvum e Ureaplasma urealyticum) e Mycoplasma hominis, nonché la determinazione della loro sensibilità agli antibiotici. Con "Mycoplasma IST" viene saggiata la sensibilità nei confronti di Doxiciclina, Eritromicina, Josamicina, Ofloxacina, Pristinamicina, Tetraciclina, mentre con "Mycoplasma IST 2", oltre a questi antibiotici, viene saggiata la sensibilità anche verso Azitromicina, Ciprofloxacina, Claritromicina.

\section{RISULTATI}

240/781 (30.7\%) dei campioni esaminati sono risultati positivi per Ureaplasma spp. Di questi, $152 / 240(63.3 \%)$ presentavano una carica $>10^{4}$ $\mathrm{UFC} / \mathrm{ml}$ e $88 / 240(36.7 \%)$ una carica $<10^{4}$ UFC/ml. Per 56/145 (38.6\%) tamponi vaginali positivi per Ureaplasma spp con carica $>10^{4}$ $\mathrm{UFC} / \mathrm{ml}$ è stata riscontrata un'associazione con altre specie microbiche (Tabella I).

In $1 / 4$ dei campioni di liquido seminale con carica $>10^{4} \mathrm{UFC} / \mathrm{ml}$ è stata riscontrata associazione con $S$. cerevisiae e $P$. mirabilis.

Riguardo all'incidenza di resistenza antibiotica, si sono osservate le variazioni seguenti: per i chinoloni, la resistenza a Ciprofloxacina da $90 \%(2003)$ a $100 \%(2007)$ e a Ofloxacina da $62 \%$ (2002) a $71 \%$ (2007); per i macrolidi, la resistenza a Eritromicina da $18 \%$ (2002) a $68 \%$ (2007), a Azitromicina da 30\% (2003) a 56\% (2007) e a Claritromicina da $0 \%$ (2003) a $23 \%$ (2007). Doxiciclina, Tetraciclina, Josamicina e Pristinamicina hanno esibito attività ottimale intorno al $100 \%$, costante in tutto 1 'arco di tempo considerato (Figura I).

Tabella I. Associazione tra Ureaplasma spp. ed altri microrganismi nel secreto vaginale

\begin{tabular}{ll}
\hline Microrganismo & $\mathbf{N}^{\circ}$ \\
\hline C. albicans & 20 \\
\hline E. coli & 12 \\
\hline C. glabrata & 4 \\
\hline E. faecalis & 4 \\
\hline S. agalactie & 4 \\
\hline G. vaginalis & 4 \\
\hline K. pneumoniae & 3 \\
\hline P. mirabilis & $\mathrm{I}$ \\
\hline E. avium & $\mathrm{I}$ \\
\hline C. krusei & $\mathrm{I}$ \\
\hline S. cerevisae & $\mathrm{I}$ \\
\hline C. lusitaniae & $\mathrm{I}$ \\
\hline
\end{tabular}

\section{CONCLUSIONI}

Il largo impiego di antibiotici di uso comune, data la necessità di trattare anche patologie urogenitali sostenute da altri microrganismi presenti in associazione, ha determinato un notevole aumento della resistenza di Ureaplasma spp. In particolare, abbiamo osservato un evidente aumento della resistenza ai fluorochinoloni tale che, nel corso dell'anno 2007, non è stato isolato nessun ceppo sensibile alla Ciprofloxacina. Importante è anche l'aumento di resistenza ad Azitromicina, considerando che questo antibiotico rappresenta un farmaco di elezione nel trattamento di patologie urogenitali sostenute da Mycoplasmi.

Evidente è stata anche la resistenza ai macrolidi di più largo uso, così come è rimasta elevata la sensibilità a Josamicina. I nostri dati, particolarmente quelli relativi agli ultimi anni di osservazione riguardo a ceppi resistenti a Tetraciclina e Doxicilina, considerati insieme ad Azitromicina farmaci di prima scelta nel trattamento delle patologie sostenute da Ureaplasma spp., sostengono la necessità dell'uso mirato degli antibiotici, anche al fine di migliorare l'approccio terapeutico empirico di queste patologie.

\section{BIBLIOGRAFIA}

1. Brunet B, de Barbeyrac B, Renaudin $\mathrm{H}$, et al. Detection of tetracycline-resistant strains of Ureaplasma urealyticum by hybridization assays. European Journal of Clinical Microbiology \& Infectious Diseases 1989; Vol. 8, N. 7: 636-638

2. Fagundo-Sierra R, Sánchez-Saínz A, Pérez-Jáuregui J. In vitro resistance of Mycoplasma hominis and Ureaplasma urealyticum clinical isolates in Mexico. Bioquimia 2006; 31 (4):124-131

3. G. Palu, S. Valisena, M. F. Barile, et al. Mechanisms of Macrolide Resistance in Ureaplasma urealyticum: A 
Study on Collection and Clinical Strains. European Journal of Epidemiology 1989; Vol. 5, No. 2: 146-153

4. H. Renaudin, C. Bébéar and J. A. Robertson In vitro susceptibility of tetracycline-resistant strains of Ureaplasma urealyticum to newer macrolides and quinolones, and a streptogramin European Journal of Clinical Microbiology \& Infectious Diseases 1991;Vol.10, N.11: 984-986

5. M.C. Roberts , G. E. Kenny. Dissemination of the tetM tetracycline resistance determinant to Ureaplasma urealyticum. Antimicrob Agents Chemother. 1986;
29(2): 350-352

6. Podda R., Consagra C., Porcu P.et al. Infezioni genitali da micoplasmi nel soggetto infertile: incidenza e sensibilità agli antibiotici. Il Patologo clinico 2003; N 9/12: 267-272

7. Xie, Xinyou, Zhang, Jun. Trends in the rates of resistance of Ureaplasma urealyticum to antibiotics and identification of the mutation site in the quinolone resistance-determining region in Chinese patients. FEMS Microbiology Letters 2006; Vol 259, N. 2: 181-186 